PROCEEDINGS OF THE

AMERICAN MATHEMATICAL SOCIETY

Volume 135, Number 10, October 2007, Pages 3187-3191

S 0002-9939(07)08885-5

Article electronically published on February 28, 2007

\title{
PERIOD TWO IMPLIES ALL PERIODS FOR A CLASS OF ODES: A MULTIVALUED MAP APPROACH
}

\author{
JAN ANDRES, TOMÁŠ FÜRST, AND KAREL PASTOR \\ (Communicated by Carmen C. Chicone)
}

\begin{abstract}
We present an elementary proof that, for a multivalued map $\varphi$ : $\mathbb{R} \multimap \mathbb{R}$ with nonempty connected values and monotone margins, the existence of a periodic orbit of any order $k>1$ implies the existence of periodic orbits of all orders. This generalizes a very recent result of this type in terms of scalar ordinary differential equations without uniqueness, due to F. Obersnel and P. Omari, obtained by means of lower and upper solutions techniques.
\end{abstract}

\section{INTRODUCTION}

In reply to a series of our papers [AFJ, AJP, AP] (cf. also [AG, Chapter III.9]) about a multivalued version of the celebrated Sharkovskii cycle coexistence theorem [S] (which is unlike the classical version applicable to scalar ordinary differential equations), F. Obersnel and P. Omari have very recently shown in $\mathrm{OO} 2$ that the existence of one subharmonid 1 implies the existence of subharmonics of all orders. Although their proof, by means of lower and upper solutions techniques, is rather simple, it relies on several technical lemmas developed earlier in OO1. Our aim here is to present an extremely simple proof of their result, in terms of multivalued maps, under the additional assumption concerning the global existence of solutions.

More precisely, the authors of $\mathrm{OO} 2$ established the following theorem for a scalar ordinary differential equation

$$
x^{\prime}=f(t, x) .
$$

Theorem 1 (OO2, Theorem 2). Let $f: \mathbb{R} \times \mathbb{R} \rightarrow \mathbb{R}$ be 1-periodic in the first variable and satisfy the $L^{1}$-Carathéodory condition $\S^{2}$ on $[0,1] \times \mathbb{R}$. If equation (11) admits a subharmonic solution of order $k>1$, then, for every $n \in \mathbb{N}=\{1,2, \ldots\}$, there exists a subharmonic solution of (11) of order $n$. In addition, the set of all subharmonic solutions of (11) of order $n$ has dimension at least $n$ as a subset of $L^{\infty}(\mathbb{R})$.

Received by the editors June 14, 2006.

2000 Mathematics Subject Classification. Primary 34A60, 34C25, 37E05, 47H04.

Key words and phrases. Periodic orbits, multivalued maps, monotone margins, Sharkovskii's theorem, ordinary differential equations without uniqueness, subharmonic solutions.

This work was supported by the Council of Czech Government (MSM 6198959214).

${ }^{1}$ Let us recall that by a subharmonic solution of order $k \in \mathbb{N}, k>1$, we understand an absolutely continuous periodic solution whose minimal period is a $k$-multiple of the minimal time period of the right-hand side of the respective equation.

${ }^{2}$ For the definition and more details, see, e.g., [F Chapter 1.1]. 
As an example of equation (11) which satisfies the assumptions of Theorem 11, the authors introduced in OO1] the equation

$$
x^{\prime}=\sqrt{|x|}-\frac{1}{8 \pi}|\arcsin (\sin (\pi t))| .
$$

In order to formulate our main result, let us recall that by the margins of a multivalued map $\varphi: \mathbb{R} \multimap \mathbb{R}$ with nonempty values (i.e., $\varphi: \mathbb{R} \rightarrow 2^{\mathbb{R}} \backslash\{\emptyset\}$ ), we understand the single-valued functions $\varphi^{*}: \mathbb{R} \rightarrow \mathbb{R} \cup\{+\infty\}$ and $\varphi_{*}: \mathbb{R} \rightarrow \mathbb{R} \cup\{-\infty\}$ defined by

$$
\begin{aligned}
& \varphi^{*}(x):=\sup \{y: y \in \varphi(x)\}, \\
& \varphi_{*}(x):=\inf \{y: y \in \varphi(x)\} .
\end{aligned}
$$

Furthermore, by a $k$-orbit of a multivalued mapping $\varphi$, we mean a sequence $\left\{x_{0}, \ldots, x_{k-1}\right\}$ such that

(i) $x_{i+1} \in \varphi\left(x_{i}\right)$, for all $i=0, \ldots, k-2, \quad x_{0} \in \varphi\left(x_{k-1}\right)$, and

(ii) the orbit is not a product orbit formed by going $p$-times around a shorter $m$-orbit, where $m p=k$.

If still

(iii) $x_{i} \neq x_{j}$, for $i \neq j ; i, j=0, \ldots, k-1$, then we speak about a primary $k$-orbit.

Theorem 2. Let $\varphi: \mathbb{R} \multimap \mathbb{R}$ be a multivalued mapping with nonempty connected values whose margins are either both nondecreasing or both nonincreasing. If $\varphi$ has a $k$-orbit with $k>1$, then $\varphi$ also has a primary $n$-orbit, for every $n \in \mathbb{N}$. Moreover, the set of all $n$-orbits (as a subset of $\mathbb{R}^{n}$ ) has dimension $n$.

We will show that Theorem 1 is a special case of Theorem 2 in terms of the Poincaré translation operator $T_{t}: \mathbb{R} \multimap \mathbb{R}$ associated with equation (1) which is defined by

$$
T_{t}(x):=\{y(t): y(\cdot) \text { is a solution of (11) with } y(0)=x\} .
$$

Hence, our note is organized in the following way. At first, we prove Theorem 2 Then we show that, under the assumption of global existence of solutions of (11), the associated Poincaré operator $T_{1}: \mathbb{R} \multimap \mathbb{R}$ satisfies the assumptions of Theorem 2 and we clarify the relationship between periodic solutions of (11) and periodic orbits of $T_{1}$. At last, we add some comments concerning the title of the present paper.

\section{Proof of Theorem 2}

Proof. Assume $\varphi^{*}, \varphi_{*}$ are nondecreasing, the other case being treated similarly. Let $\left\{x_{0}, \ldots, x_{k-1}\right\}$ be the considered $k$-orbit. Redenote the points as $\left\{y_{0}, \ldots, y_{k-1}\right\}$ so that $y_{0}$ is the minimal element. Observe that there exists $y_{i} \in\left\{y_{0}, \ldots, y_{k-1}\right\}$ such that $y_{i}>y_{0}$ and $y_{0} \in \varphi\left(y_{i}\right)$ (otherwise, the $k$-orbit would be reduced to the single point $\left.y_{0}\right)$. The monotonicity of $\varphi_{*}$ now implies that $y_{0} \in \varphi\left(y_{0}\right)$. Observe further that due to the connectedness of the values of $\varphi$, the set $\varphi\left(y_{0}\right)$ contains a nondegenerate interval, say $\left[y_{0}, a\right]$ (otherwise, the $k$-orbit would again be reduced to the single point $\left.y_{0}\right)$. We may take $a<y_{i}$. Summing up the above arguments, the whole rectangle $\left[y_{0}, a\right]^{2}$ is contained in the graph $\Gamma_{\varphi}$ of $\varphi$ because of the monotonicity of the margins of $\varphi$. 


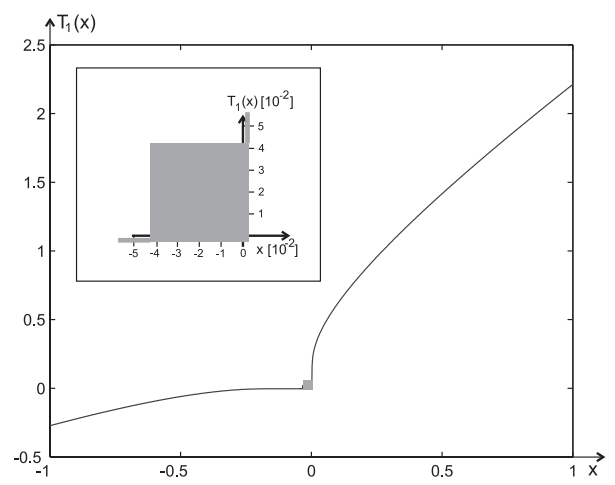

Figure 1. Poincaré operator $T_{1}$ for equation (2). The figure was generated using MatLab.

Now, choose points $z_{0}, \ldots, z_{n-1}$ such that, e.g.,

$$
y_{0}=z_{0}<z_{1}<\cdots<z_{n-2}<z_{n-1}=a .
$$

The set $\left\{z_{0}, \ldots, z_{n-1}\right\}$ is then the desired primary $n$-orbit.

Notice that, in fact, any $n$-tuple $\left\{z_{0}, \ldots z_{n-1}\right\}$ with $y_{0} \leq z_{i} \leq a$, for $i=0, \ldots n-$ 1 , which satisfies the above condition (ii) is an $n$-orbit of $\varphi$. Therefore, the set of all such $n$-tuples has dimension $n$.

Remark 1. The Poincaré operator associated to equation (2) is depicted in Figure 1.

\section{Relationship of Theorems 1 And 2}

In order to demonstrate that Theorem 1 follows, under the assumption of global existence of solutions of (1), from the conclusion of Theorem 2, we need to show that

(i) $T_{1}$ has nonempty and connected values,

(ii) the margins $T_{1}^{*}$ and $T_{1 *}$ of $T_{1}$ are nondecreasing,

(iii) each $k$-periodic orbit of $T_{1}$ determines a set of $k$-periodic solutions of (1), and the sets of $k$-periodic solutions determined by different $k$-orbits are disjoint.

Condition (i) follows directly from the well-known Kneser theorem (cf., e.g., F, Theorem 5, Chapter 1.1]) saying that, under the assumptions of Theorem 1, the values $T_{1}(x)$ are continua (i.e., nonempty compact and connected sets), for every $x \in \mathbb{R}$.

As concerns condition (ii), take $x_{1}<x_{2}$, denote $y_{i}:=T_{1}^{*}\left(x_{i}\right)$, for $i=1,2$, and suppose, on the contrary, that $y_{1}>y_{2}$. There exist solutions $z_{i}:[0,1] \rightarrow \mathbb{R}$ with $z_{i}(0)=x_{i}$ and $z_{i}(1)=y_{i}$, for $i=1,2$. The solutions $z_{1}$ and $z_{2}$ necessarily cross each other at some point $t=t_{0} \in(0,1)$. But then the function $\tilde{z}:[0,1] \rightarrow \mathbb{R}$ defined by

$$
\tilde{z}(s):= \begin{cases}z_{2}(s), & \text { for } s \leq t_{0} \\ z_{1}(s), & \text { for } s \geq t_{0}\end{cases}
$$

is also a solution, and consequently $y_{1} \in T_{1}\left(x_{2}\right)$ which is a contradiction with $y_{2}$ being the maximal element of $T_{1}\left(x_{2}\right)$. We can show analogously that $T_{1 *}$ is also nondecreasing. 
To conclude by (iii), let us realize that if $\left\{x_{0}, \ldots, x_{k-1}\right\}$ is a $k$-orbit of $T_{1}$, then any solution of (1) with $x(0)=x_{0}, \ldots, x(k-1)=x_{k-1}$ and $x(k)=x_{0}$ becomes, after a $k$-periodic prolongation, $k$-periodic. Obviously, if two $k$-orbits of $T_{1}$ differ, e.g., in $x_{i}$, then the corresponding $k$-periodic solutions differ at $t=i 3$

Let us further indicate that the dimension of the set of all $n$-periodic solutions of (11) (as a subset of $L^{\infty}(\mathbb{R})$ ) is at least $n$ and that it also follows from Theorem 2 , Consider the operator $T_{1}$ again and notice (in view of the proof of Theorem 2) that $\left[y_{1}, a\right] \subset T_{1}\left(\left[y_{1}, a\right]\right)$. Thus, there exist solutions $\alpha, \beta:[0,1] \rightarrow \mathbb{R}$ such that

$$
\alpha(0)=y_{1}, \quad \alpha(1)=a \quad \text { and } \quad \beta(0)=a, \quad \beta(1)=y_{1} .
$$

Now, we can proceed exactly in the same way as in the proof of [0O2, Theorem 2] to make the conclusion about the dimension of the set of $n$-periodic solutions.

\section{Concluding Remarks}

We could see that, via the Poincaré operators, Theorem 2 is indeed a significant generalization of Theorem 1. Mapping $\varphi$ in Theorem 2 need not be (besides other assumptions) upper semicontinuous, while the Poincaré mapping $T_{1}$ related to Theorem 1 must be so (cf. AG, Chapter III.4]). Moreover, the proof of Theorem 2 is elementary and without technicalities. On the other hand, unlike in Theorem 1 , the global existence of solutions of (11) has to be assumed.

Let us finally make several comments to the title borrowed from $\mathrm{OO} 2$. One can readily check that, in the case of uniqueness, the single-valued Poincaré operator is (strictly) increasing, and so no periodic orbit can occur. Thus, if equation (11) has a 2-periodic solution, $T_{1}$ admits a 2 -orbit, and subsequently (1) is not uniquely solvable. This already implies the existence of local dynamical instabilities, i.e., the sensitive dependence on initial conditions. The conclusions of Theorems 11 and 2 are, however, much deeper, namely that (unlike the version of Sharkovskii's theorem developed in AFJ, AJP, AP ) the existence of just one subharmonic implies the existence of large sets of subharmonics of all orders coexisting with very complicated dynamics (whence the slight modification of our title when compared to [002]). This complexity led F. Obersnel and P. Omari to their title of $\mathrm{OO} 2$.

The notion of deterministic chaos has been, however, so far related exclusively to single-valued maps. Although it can easily be generalized to multivalued maps, such an extension would not be proper, as can be seen from Figure 1. For instance, a multivalued constant (the black square in Figure 1) can be easily checked to be chaotic in the sense of Devaney, because, besides the mentioned sensitive dependence on initial conditions (typical for chaos in the sense of Li-Yorke), it exhibits transitivity and density of periodic orbits on an invariant set. A much more appropriate notion of chaos for multivalued maps would therefore be the one for induced hyperspace maps which already become single-valued and which we call hyperchaos (cf. $\mathrm{AS}$ ). This is, however, another story.

\section{REFERENCES}

[AFJ] J. Andres, J. Fišer and L. Jüttner: On a multivalued version of the Sharkovskii theorem and its application to differential inclusions, Set-Valued Anal. 10 (2002), 1-14. MR1888453 (2002m:37057)

\footnotetext{
${ }^{3}$ In fact, any $k$-orbit of $T_{1}$ determines, in general, many $k$-periodic solutions of (1), so there is no one-to-one correspondence between $k$-orbits of $T_{1}$ and $k$-periodic solutions of (1).
} 
[AG] J. Andres and L. Górniewicz: Topological Fixed Point Principles for Boundary Value Problems, Kluwer, Dordrecht, 2003. MR1998968(2005a:47102)

[AJP] J. Andres, L. Jüttner and K. Pastor: On a multivalued version of the Sharkovskii theorem and its application to differential inclusions, II, Set-Valued Anal. 13 (2005), 47-68. MR2128697(2006c:37018)

[AP] J. Andres and K. Pastor: A version of Sharkovskii's theorem for differential equations, Proc. Amer. Math. Soc, 133 (2005), 449-453. MR2093067 (2005e:34124)

[AS] J. Andres and P. Šnyrychová: Hyperchaos induced by multivalued maps, in preparation.

[F] A.F. Filippov: Differential Equations with Discontinuous Right-Hand Sides, Kluwer, Dordrecht, 1988. MR1028776 (90i:34002)

[LY] T.-Y. Li and J.A. Yorke: Period three implies chaos, Amer. Math. Monthly 82 (1975), 985-992. MR0385028 (52:5898)

[OO1] F. Obersnel and P. Omari: Old and new results for first order periodic ODEs without uniqueness: a comprehensive study by lower and upper solutions, Adv. Nonlin. Stud. 4 (2004), 323-376. MR2079818 (2005g:34093)

[OO2] F. Obersnel and P. Omari: Period two implies chaos for a class of ODEs, Proc. Amer. Math. Soc., posted on January 9, 2007, PII: 5002-9937(07)08700-X (to appear in print).

[S] A.N. Sharkovskii: Coexistence of cycles of a continuous map of a line into itself, Ukrain. Math. J. 16(1964), 61-71 (Russian); English translation: Int. J. Bifurc. Chaos. 5 (1995), 1263-1273. MR1361914 (96j:58058)

Department of Mathematical Analysis and Applications of Mathematics, Faculty of Science, Palacký University, Tomkova 40, 77900 Olomouc-Hejčín, Czech Republic

E-mail address: andres@inf.upol.cz

Department of Mathematical Analysis and Applications of Mathematics, Faculty of Science, Palacký University, Tomkova 40, 77900 Olomouc-Hejčín, Czech Republic

E-mail address: tomas.furst@seznam.cz

Department of Mathematical Analysis and Applications of Mathematics, Faculty of Science, Palacký University, Tomkova 40, 77900 Olomouc-Hejčín, Czech Republic

E-mail address: pastor@inf.upol.cz 